\title{
Stationary Beam Radiation Therapy
}

National Cancer Institute

\section{Source}

National Cancer Institute. Stationary Beam Radiation Therapy. NCI Thesaurus. Code C60646.

Radiation therapy without displacement of one or more mechanical axes relative to the patient during irradiation. 